Pacific Journal of Mathematic 


\title{
HOMOTOPY DIMENSION OF SOME ORBIT SPACES
}

\author{
Vo ThaNH LIEM
}

The homotopy dimension of a compact absolute neighborhood retract space $X$ is defined to be the least dimension among all the finite $\mathrm{CW}$-complexes which have the same homotopy type of $X$. We show that actions of finite groups or actions of tori (with finite orbit types) on a finite-dimensional compact absolute neighborhood retract $X$ do not raise homotopy dimension if the homotopy dimension of $X$ is not two.

1. Introduction and preliminaries. Through this note, all actions are of finite types.

In [7], Oliver gave an affirmative answer to Conner's conjecture: "The orbit space of an action of a compact Lie group on a finitedimensional AR is an AR". From West [10], it follows that every compact absolute neighborhood retract $X$ (CANR $X$ ) has the homotopy type of a finite complex. So, we can define the homotopy dimension (h.d.) of a CANR $X$ by

h.d. $(X)=\min \{\operatorname{dim} K \mid K$ is a finite complex and $K \cong X\}$.

On the other hand, Conner [5] has shown that the orbit space of an action of a compact Lie group on a finite-dimensional CANR is a CANR. It is natural to wonder whether the actions of a compact Lie group on a CANR can raise the homotopy dimension. We will show that the homotopy dimension does not increase when h.d. $(X) \neq 2$ and when the action comes from either a finite group or a toral group.

Combining a well-known result of Wall (Thm. F, [8]) and the result of West [10] (mentioned above), we can easily obtain the following lemma that will be needed in the sequel.

LEMma 0. A CANR has the homotopy type of a k-dimensional finite complex if and only if $H_{q}(\tilde{X} ; Z)=0$ for all $q>k$ and $H^{k+1}(X ; \beta)=0$ for every coefficient bundle $\beta$ of $Z \pi_{1}(X)$-modules over $X$ if $k \neq 2$. Moreover, if $H_{q}(\widetilde{X} ; Z)=0$ for $q>2$ and $H^{3}(X ; \beta)=0$; then h.d. $(X) \leqq 3$.

2. Orbits of action of finite groups. Let $G$ be a cyclic group of order $p$ with a genertor $g$. The notation in [1] will be used as follows $1-g$ and $1+g+\cdots+g^{p-1}$ will be denoted respectively by $\tau$ and $\sigma$. If one of these is denoted by $\rho$, the other will be denoted 
by $\bar{\rho}$. If $\beta$ is a sheaf of $Z_{p}$-modules over $X / G$, let $\underline{A}$ denote the sheaf

$$
\left\{H^{0}\left(\pi^{-1} y ; \pi^{*} \beta \mid \pi^{-1} y\right) \mid y \in X / G\right\} \text { over } X / G,
$$

where $\pi^{*} \beta$ is the pull back of $\beta$ associated with the orbit map $\pi: X \rightarrow X / G$. If $U$ is an open subset of $X / G$, let $\underline{A}_{U}$ denote the sheaf

$$
\left[\cup\left\{H^{0}\left(\pi^{-1} y ; \pi^{*} \beta \mid \pi^{-1} y\right) \mid y \in U\right\}\right] \cup\left[\left\{0_{y} \mid y \in X / G\right\}\right]
$$

and let $\underline{A}_{F}(F$ closed in $X / G)$ denote $\underline{A} / \underline{A}_{(X / G)-F}$ (refer to page 41 of [1]).

It will be convenient to establish the following preliminary lemmas before we begin the proof of the main result.

LeMma 1. Let $G=Z_{p}, p$ prime, act on a CANR $X$ with fixed point set $F$. Assume that $m=\operatorname{dim} X<\infty$ and that $\beta_{p}$ is a bundle of coefficients of $Z_{p} \pi_{1}(X / G)$ - modules over $X / G$. If h.d. $(X) \leqq k$, then $H^{q}\left(X / G ; \beta_{p}\right)=0$ for all $q \geqq k+1$.

Proof. Think of $\rho$ and $\bar{\rho}$ as endomorphisms of the sheaf $\underline{A}$ and denote their images respectively by $\rho \underline{A}$ and $\bar{\rho} \underline{A}$. Since $Z_{p}$ is a field, it follows that the following sequence of sheaves over $X / G$

$$
0 \longrightarrow \underline{\rho} \underline{A} \longrightarrow \underline{A} \stackrel{\rho \oplus \eta}{\longrightarrow} \rho \underline{A} \oplus \underline{A}_{F} \longrightarrow 0
$$

is exact, where $\bar{\rho} \underline{A} \rightarrow \underline{A}$ is the inclusion and where $\eta: \underline{A} \rightarrow \underline{A}_{F}$ is the quotient homomorphism (Lemma 4.1 of [1]). This sequence induces an exact cohomology sequence

$$
\begin{aligned}
\cdots & \longrightarrow H^{n}(X / G ; \bar{\rho} \underline{A}) \longrightarrow H^{n}(X / G ; \underline{A}) \\
& \longrightarrow H^{n}(H / G ; \rho \underline{A}) \oplus H^{n}\left(X / G ; \underline{A}_{F}\right) \longrightarrow \cdots .
\end{aligned}
$$

Let $H^{n}(\rho)$ denote $H^{n}(X / G ; \rho \underline{A})$. Observe $H^{n}\left(X / G ; \underline{A}_{F}\right)=H^{n}(F$; $\left.\beta_{p} \mid F\right)$; then, from the above cohomology sequence and the fact that $H^{n}(X / G ; \underline{A}) \cong H^{n}\left(X ; \pi^{*} \beta_{p}\right)$ (see page $\left.35,[1]\right)$, there are the following exact sequences:

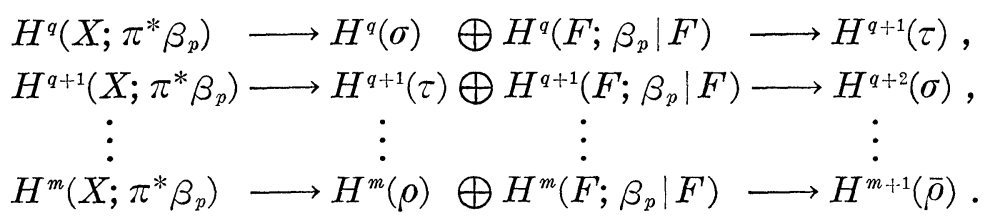

Since h.d. $(X) \leqq k$, it follows from Lemma 0 that $H^{n}\left(X, \pi^{*} \beta_{p}\right)=0$, for all $n \geqq q \geqq k+1$. On the other hand, $H^{m+1}(\bar{\rho})=0$ since $\operatorname{dim} X=m<\infty$. Thus, we can show inductively that

(1) $H^{q}\left(X / G, F ; \beta_{p}\right)=H^{q}(\sigma)=0$, and

(2) $H^{q}\left(F ; \beta_{p} \mid F\right)=0$. 
Hence, from the exact sequence of the pair $(X / G, F)$,

$$
\cdots \longrightarrow H^{q}\left(X / G, F ; \beta_{p}\right) \longrightarrow H^{q}\left(X / G ; \beta_{p}\right) \longrightarrow H^{q}\left(F ; \beta_{p} \mid F\right) \longrightarrow \cdots,
$$

it follows that $H^{q}\left(X / G ; \beta_{p}\right)=0$; and the proof of lemma is complete.

Lemma 2. Let $G=Z_{p}, p$ prime, act on a CANR $X$ with fixed point set $F \neq \varnothing$. Assume that $\operatorname{dim} X=m<\infty$ and that $\beta$ is a bundle of coefficients of $Z \pi_{1}$-modules over $X / G$. Then $H^{q}(X / G ; \beta)=0$ for all $q \geqq k+1$, if h.d. $(X) \leqq k$.

Proof. Consider the following diagram



where $\mu^{*}$ is the transfer map [1] and where the horizontal exact sequence is from the exact sequence of bundles of coefficients over $X / G$ :

$$
0 \longrightarrow \beta \stackrel{\times p}{\longrightarrow} \beta \longrightarrow \beta_{p} \longrightarrow 0 \text {. }
$$

So, it follows easily that $H^{q}(X / G ; \beta)=0$ if $q \geqq k+1$, since $H^{q}(X$; $\left.\pi^{*} \beta\right)=0$ by Lemma 0 and $H^{q}\left(X / G ; \beta_{p}\right)=0$ by Lemma 1 . The proof is now complete.

Lemma 3. Let a finite group $G$ act on $X$ with fixed point set $F \neq \varnothing$. If $X$ has the homotopy type of a simplicial complex $K^{k}$, then $H_{q}(\widetilde{X / G} ; Z)=0$ for all $q>k$.

Proof. Let $\pi^{*}(\widetilde{X / G})$ be the pullback of the universal covering space $p: \widetilde{X / G} \rightarrow X / G$ associated with the orbit map $\pi: X \rightarrow X / G$. Then, the induced map $\bar{P}: \pi^{*}(\widetilde{X / G}) \rightarrow X$ is a covering map and the lifting map $\pi^{*}$ of $\pi$ is the orbit map of the induced action of $G$ on $\pi^{*}(\overparen{X / G})$. Now, since $X \cong K^{k}$, it follows that $H_{q}\left(\pi^{*}(\widetilde{X / G}), Z\right)=0$ for $q \geqq k+1$. Then, the Smith theorem in the integral homology theory shows that $H_{q}(\widetilde{X / G}, Z)=0$ for $q \geqq k+1$. (Similar to the proof of Lemma 2 above by use of the transfer map $\mu_{*}$ on page 119 of [3].) Hence, the proof is complete.

THeOREM 1. Suppose that a finite group $G$ acts on a finite dimensional CANR $X$. If h.d. $(X) \leqq k$ and $k \neq 2$, then h.d. $(X / G) \leqq k$. If $k=2$, h.d. $(X / G \leqq 3)$. 
Proof. Step 1. $G=Z_{p}, p$ prime.

Case 1. $F=\varnothing$. See Lemma 2 of [6].

Case 2. $F \neq \varnothing$. It follows from Lemma 2 and Lemma 3 above that

(1) $H^{q}(X / G ; \beta)=0, q \geqq k+1$ and for any bundle coefficient $\beta$ over $X / G$,

(2) $H_{q}(X / G ; Z)=0, q \geqq k+1$.

So, it follows from Lemma 0 that h.d. $(X) \leqq k$.

Step 2. $G$ is cyclic of order $p^{n}, p$ prime. We prove inductively on $|G|$, the order of $G$. Let $H$ be a subgroup of $G$ of order $p^{n-1}$ then, h.d. $(X / H) \leqq k$ by induction hypothesis and the proof is complete by Step 1.

Step 3. $G$ is a finite p-group. First, by an inductive proof as in Step 2 we may assume that $G$ is abelian, since $G$ is solvable. Therefore, we can write $G=Z_{p}^{n_{1}} \oplus \cdots \oplus Z_{p}^{n_{k}}$. Then, again an inductive proof as above will complete the proof for this case.

Step 4. General case. The proof will be similar to that of Theorem III. 5.2 in [1].

Suppose that $|G|=p_{1}^{n_{1}} \cdots p_{s}^{n_{s}}$ and that $K_{j}$ is a $p_{j}$-Sylow subgroups of $G$, and denote $\pi_{2, j}$ the canonical map $X / K_{j} \rightarrow X / G$ for $j=1,2, \cdots, s$ as in [1]. Define $\pi^{\prime}: H^{*}(X / G ; \beta) \rightarrow \sum_{j=1}^{s} H^{*}\left(X / K_{j} ; \pi_{2}^{*} \beta\right)$ by

$$
\pi^{\prime}=\pi_{2,1}^{*}+\cdots+\pi_{2, s}^{*} .
$$

Observe that $H^{q}\left(X / K_{j} ; \pi^{*} \beta\right)=0$ for $q \geqq k+1$ and $j=1,2, \cdots, s$ by Step 3 above. Hence, if we can show that $\pi^{\prime}$ is injective, then $H^{q}(X / G ; \beta)=0$ for $q \geqq k+1$. Therefore, the theorem will follow by Lemma 0 and Lemma 3 above.

Now, let $\mu_{j}^{\prime}: H^{*}\left(X / K_{j} ; \pi_{2, j}^{*} \beta\right) \rightarrow H^{*}(K / G ; \beta)$ be the tranfer map [1] such that $\mu_{j}^{\prime} \pi_{2, j}^{*}$ is the multiplication by $|G| /\left|K_{j}\right|$. If $r \in \operatorname{Ker} \pi^{\prime}$, then we have $\left.|G| /\left|K_{j}\right|\right) \cdot r=\mu_{j}^{\prime} \pi_{2, j}^{*}(r)=0$ for each $j=1,2, \cdots, s$, since $\pi_{2, j}^{*}=0$. Therefore, for each $j=1,2, \cdots, s$

$$
\left(p_{1}^{n_{1}} \cdots p_{j-1}^{n_{j-1}} p_{j+1}^{n_{j+1}} \cdots p_{s}^{n_{s}}\right) \cdot r=0 .
$$

Since the family $p_{1}^{n_{1}} \cdots p_{j+1}^{n_{j-1}} p_{j+1}^{n_{j+1}} \cdots p_{s}^{n_{s}}, j=1,2, \cdots, s$, is relatively prime, it follows that $r=0$, and the proof is now complete.

\section{Orbits of actions of total groups.}

Lemma 4. Suppose that the circle group $S^{1}$ acts on a finite- 
dimensional CANR $X$. If h.d. $(X) \leqq k$, then $H^{q}\left(X / S^{1} ; \beta\right)=0$ for all $q \geqq k+1$ and for all bundles of coefficients $\beta$ over $X / S^{1}$.

Proof. Assume that $H_{1}, \cdots, H_{s}$ are finite isotropy subgroups of the action. Let $G$ be a finite cyclic subgroup of $S^{1}$ such that $H_{1}, \cdots, H_{s}$ are subgroups of $G$. Then h.d. $(X / G) \leqq k$ by the theorem above. So, we may assume that the action is semi-free, i.e., it has only two orbit types $\{e\}$ and $S^{1}$. Let $\beta$ be a bundle of coefficients of $Z \pi_{1}$-modules over $X / S^{1}$, where $\pi_{1}=\pi_{1}\left(X / S^{1}\right)$. From Lemma 0 , it follows that $H^{q}\left(X ; \pi^{*} \beta\right)=0$ for all $q \geqq k+1$, where $\pi: X \rightarrow X / S^{1}$ is the orbit map.

Case 1. $F=\varnothing$. Since the action is free, $\left\{H^{0}\left(\pi^{-1} y ; \pi^{*} \beta\right): y \in\right.$ $\left.X / S^{1}\right\}=\beta$ and $\left\{H^{1}\left(\pi^{-1} y ; \pi^{*} \beta\right): y \in X / S^{1}\right\}=\beta$. An observations on Leray spectral sequence (as in Case 2 ) proves the lemma for this case.

Case 2. $F \neq \varnothing$. Since $\pi^{-1}(y)=\{e\}$ or $S^{1}$, we have

(1) $E_{2}^{q, 0}=H^{q}\left(X / S^{1} ; H^{0}\left(\pi^{-1} y ; \pi^{*} \beta \mid \pi^{-1} y\right)\right)=H^{q}\left(X / S^{1} ; \beta\right)$,

(2) $E_{2}^{q, 1}=H^{q}\left(X / S^{1} ; H^{1}\left(\pi^{-1} y ; \pi^{*} \beta \mid \pi^{-1} y\right)\right)=H^{q}\left(X / S^{1}, F ; \beta\right)$, and

(3) $\quad E_{2}^{q, s}=0$ if $s \geqq 2$.

We now proceed by induction on $q$. Since $\operatorname{dim} X<\infty$, we may assume that $H^{q}\left(X / S^{1} ; \beta\right)=0$ for $q \geqq k+2$, then we will show that $H^{k+1}\left(X / S^{1} ; \beta\right)=0$.

Step 1. To show that $H^{q}\left(X / S^{1}, F ; \beta\right)=0$ for $q \geqq k+1$. By the induction hypothesis, we observe that for each $q \geqq k+2$, the $E_{2}$ term, $E_{2}^{q, 0}$, of the Leray spectral sequence for the map $\pi$ (page 140, [2]) is trivial, since $E_{2}^{q, 0}=H^{q}\left(X / S^{1} ; \beta\right)$ by (1). Observing the Leray spectral sequence $\left\{E_{2}^{q, s}\right\}$ of $\pi$, we can show that for all $r \geqq 2$

(a) $E_{r}^{k+1,1}=E_{2}^{k+1,1}$,

and

(b) $E_{r}^{k+2,0}=0$;

therefore,

(a ( $\left.^{1}\right) \quad E_{\infty}^{k+1,1}=H^{k+1}\left(X / S^{1}, F ; \beta\right)$ by $(2)$,

and

(b) $\quad E_{\infty}^{k+2,0}=0$.

Now, from the convergence of $\left\{E_{2}^{q, s}\right\}$ to $H^{*}\left(X ; \pi^{*} \beta\right)$ and from the fact that $H^{k+2}\left(X ; \pi^{*} \beta\right)=0$ by Lemma 0 , we can show that $H^{k+1}\left(X / S^{1}, F ; \beta\right)=0$.

Step 2. To show that $H^{q}\left(X, F ; \pi^{*} \beta\right)=0$ for $q \geqq k+2$. Consider the Leray spectral sequence (page 140, [2]) of the map of pairs $\pi:(X, F) \rightarrow\left(X / S^{1}, F\right)$. First we observe that the sheaf $\xi=$ $\left\{H^{0}\left(\pi^{-1} y, \pi^{-1}(y \cap F) ; \pi^{*} \beta \mid \pi^{-1} y\right) \mid y \in X / S^{1}\right\}$ and the sheaf $\eta=\left\{H^{1}\left(\pi^{-1} y\right.\right.$, 
$\left.\left.\pi^{-1}(y \cap F) ; \pi^{*} \beta \mid \pi^{-1} y\right) \mid y \in X / S^{1}\right\}$ are the same over $X / S^{1}$, since $\pi^{-1}(y)=$ $\{e\}$ or $S^{1}$. Moreover, from the definition of the relative cohomology (Prop. II. 12.2, [2]), it follows that $H^{*}\left(X / S^{1}, F ; \beta\right)=H^{*}\left(X / S^{1} ; \xi\right)$. Then, from Step 1 it follows that

$$
E_{2}^{q, s}= \begin{cases}H^{q}\left(X / S^{1}, F ; \beta\right)=0 & \text { if } q \geqq k+1 \\ 0 & \text { if } s \geqq 2 .\end{cases}
$$

Therefore, $E_{\infty}^{q s}=0$ when $q+s \geqq k+2$. Consequently, for $q \geqq k+2$ $H^{q}(X, F ; \beta)=0$, since $\left\{E_{2}^{q, s}\right\}$ converges to $H^{*}(X, F ; \beta)$.

Step 3. To show that $H^{q}\left(X / S^{1} ; \beta\right)=0$ for $q \geqq k+1$. First, from the exact cohomology sequence of the pair $(X, F)$ and from the fact of $H^{q}\left(X, F ; \pi^{*} \beta\right)=0$ for $q \geqq k+2$, it follows that $H^{q}\left(F ; \pi^{*} \beta \mid F\right)=0$ for $q \geqq k+1$. Then, we observe that $H^{*}\left(F ; \pi^{*} \beta \mid F\right)=H^{*}(F ; \beta \mid F)$, since $F$ is the fixed point set. So, $H^{q}(F ; \beta \mid F)=0$ for $q \geqq k+1$. Therefore, the exactness of the cohomology sequence of the pair $\left(X / S^{1}, F\right)$ shows that $H^{q}\left(X / S^{1} ; \beta\right)=0$ for $q \geqq k+1$, since $H^{q}\left(X / S^{1}\right.$, $F ; \beta)=0$ by Step 1 , and the proof of lemma is now complete.

THEOREM 2. Suppose that $T^{m}$ acts on a finite-dimensional CANR $X$. Then and

(1) h.d. $\left(X / T^{m}\right) \leqq$ h.d. (X) if h.d. $(X) \neq 2$,

(2) h.d. $\left(X / T^{m}\right) \leqq 3$ if h.d. $(X)=2$.

Proof. By induction $m$, without loss of generality we only consider the actions of $S^{1}$. By Lemmas 0 and 4, we only have to show that $H_{q}\left(\widetilde{X / S^{1}} ; Z\right)=0$ for all $q \geqq k+1$. Again, by Lemma 4 above, $H^{q}\left(\overparen{X / S^{1}} ; Z\right)=0$ for all $q \geqq k+1$; therefore $\operatorname{Ext}\left(H_{q-1}\left(\widetilde{X / S^{1}}\right) ; Z\right)=0$ and $\operatorname{Hom}\left(H_{q}\left(\widetilde{X / S^{1}} ; Z\right) ; Z\right)=0$ for all $q \geqq k+1$ by the universalcoefficient theorem (Thm. 5.5.3 in [8]). Hence, for each $q \geqq k+1$ $\operatorname{Ext}\left(H_{q}\left(\widetilde{X / S^{1}} ; Z\right) ; Z\right)=0$ and Hom $\left(H_{q}\left(\widetilde{X / S^{1}} ; Z\right) ; Z\right)=0$; and it follows from Theorem V.13.7 in [2] that $H_{q}\left({\widetilde{X / S^{1}}}^{1} ; Z\right)=0$. The proof is now complete.

COROLlaRY. Let $G$ be a compact Lie group such that $\left|G / G_{0}\right|$ is finite, where $G_{0}$ is the torus identity component of $G$. Let $G$ act on a finite-dimensional CANR $X$. Then,

(1) if h.d. $(X) \neq 2$, then h.d. $(X / G) \leqq$ h.d. $(X)$,

(2) if h.d. $(X)=2$, then h.d. $(X / G) \leqq 3$.

We conclude this paper by some remarks. 
REMARKS. (1) It is a well-known problem in infinite-dimensional topology to determine whether the orbit space of an action of compact Lie group on the Hilbert cube $\Pi_{1}^{\infty}[0,1]$ is a CAR. This explains (maybe) the condition $\operatorname{dim} X<\infty$ in the above statements.

(2) The limitation, when h.d. $(X)=2$, is from an unsettled problem.

(3) The author does not see how to extend these results for the case of actions of compact Lie groups on a CANR.

\section{REFERENCES}

1. A. Borel, Seminar on Transformation groups, Princeton University Press, \#46, 1960.

2. G. E. Bredon, Sheaf Theory, McGraw-Hill, 1967.

3. - Introduction to Compact Transformation Groups, Academic Press, 1972.

4. T. A. Chapman, Lecture on Hilbert cube manifolds, CBMS Regional conference series in Math., $\$ 28$, AMS, 1975.

5. P. E. Conner, Retraction properties of the orbit spaces of a compact topological transformation group, Duke Math. J., 27 (1960), 341-357.

6. V. T. Liem, Factorization of free actions of finite groups on compact Q-manifolds, Proc. of Amer. Math. Soc., 75 (1979), 334-338.

7. R. Oliver, A proof of the Conner conjecture, Ann. of Math., 103 (1976), 637-644.

8. E. H. Spanier, Algebraic Topology, McGraw Hill 1966.

9. C. T. C. Wall, Finiteness conditions for CW-complexes, Ann. of Math., (2) 81 (1965), 55-69.

10. J. E. West, Mapping Hilbert cube manifolds to ANR's: A solution of a conjecture of Borsuk, Ann. of Math., 106 (1977), 1-18.

Received November 30, 1979.

University of Alabama

University, AL 35486 



\section{PACIFIC JOURNAL OF MATHEMATICS}

\section{EDITORS}

DONALD BABBITT (Managing Editor)

University of California

Los Angeles, CA 90024

HUGo RossI

University of Utah

Salt Lake City, UT 84112

C. C. MOORE and ANDREW OGG

University of California

Berkeley, CA 94720
J. DUGUNDJI

Department of Mathematics

University of Southern California

Los Angeles, CA 90007

R. FinN and J. Milgram

Stanford University

Stanford, CA 94305

\section{ASSOCIATE EDITORS}
R. ARENS
E. F. BECKENBACH
B. H. NeumanN
F. WOLF
K. Yoshida

\section{SUPPORTING INSTITUTIONS}

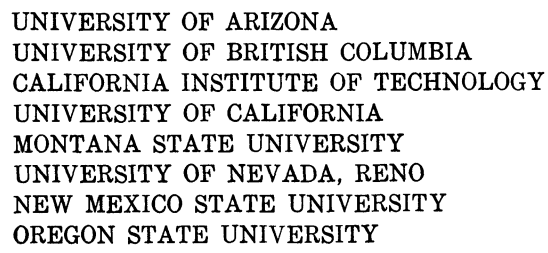

UNIVERSITY OF ARIZONA

UNIVERSITY OF BRITISH COLUMBIA CALIFORNIA INSTITUTE OF TECHNOLOGY

UNIVERSITY OF CALIFORNIA

MONTANA STATE UNIVERSITY

UNIVERSITY OF NEVADA, RENO

NEW MEXICO STATE UNIVERSITY

OREGON STATE UNIVERSITY

\author{
UNIVERSITY OF OREGON \\ UNIVERSITY OF SOUTHERN CALIFORNIA \\ STANFORD UNIVERSITY \\ UNIVERSITY OF HAWAII \\ UNIVERSITY OF TOKYO \\ UNIVERSITY OF UTAH \\ WASHINGTON STATE UNIVERSITY \\ UNIVERSITY OF WASHINGTON
}

The Supporting Institutions listed above contribute to the cost of publication of this Journal, but they are not owners or publishers and have no responsibility for its content or policies.

Mathematical papers intended for publication in the Pacific Journal of Mathematics should be in typed form or offset-reproduced, (not dittoed), double spaced with large margins. Please do not use built up fractions in the text of the manuscript. However, you may use them in the displayed equations. Underline Greek letters in red, German in green, and script in blue. The first paragraph or two must be capable of being used separately as a synopsis of the entire paper. Please propose a heading for the odd numbered pages of less than 35 characters. Manuscripts, in triplicate, may be sent to any one of the editors. Please classify according to the scheme of Math. Reviews, Index to Vol. 39. Supply name and address of author to whom proofs should be sent. All other communications should be addressed to the managing editor, or Elaine Barth, University of California, Los Angeles, California, 90024.

50 reprints to each author are provided free for each article, only if page charges have been substantially paid. Additional copies may be obtained at cost in multiples of 50 .

The Pacific Journal of Mathematics is issued monthly as of January 1966. Regular subscription rate: $\$ 102.00$ a year (6 Vols., 12 issues). Special rate: $\$ 51.00$ a year to individual members of supporting institutions.

Subscriptions, orders for numbers issued in the last three calendar years, and changes of address shoud be sent to Pacific Journal of Mathematics, P.O. Box 969, Carmel Valley, CA 93924, U.S.A Old back numbers obtainable from Kraus Periodicals Co., Route 100, Millwood, NY 10546.

\section{PUBLISHED BY PACIFIC JOURNAL OF MATHEMATICS, A NON-PROFIT CORPORATION}

Printed at Kokusai Bunken Insatsusha (International Academic Printing Co., Ltd.). 8-8, 3-chome, Takadanobaba, Shinjuku-ku, Tokyo 160, Japan. 


\section{Pacific Journal of Mathematics}

Vol. 92, No. $2 \quad$ February, 1981

Bruce Allem Anderson and Philip A. Leonard, Sequencings and Howell designs

Kevin T. Andrews, Representation of compact and weakly compact

operators on the space of Bochner integrable functions . . . . . . . . 257

James Glenn Brookshear, On the structure of hyper-real $z$-ultrafilters . . . . . 269

Frank John Forelli, Jr., A necessary condition on the extreme points of a class of holomorphic functions. II ...................... 277

Richard J. Friedlander, Basil Gordon and Peter Tannenbaum, Partitions of groups and complete mappings ......................... 283

Emden Robert Gansner, Matrix correspondences of plane partitions ......295

David Andrew Gay and William Yslas Vélez, The torsion group of a radical extension ..........................................

André (Piotrowsky) De Korvin and C. E. Roberts, Convergence theorems for some scalar valued integrals when the measure is Nemytskii ...... 329

Takaŝi Kusano and Manabu Naito, Oscillation criteria for general linear ordinary differential equations $\ldots \ldots \ldots \ldots \ldots \ldots \ldots \ldots \ldots \ldots \ldots \ldots \ldots \ldots \ldots \ldots$

Vo Thanh Liem, Homotopy dimension of some orbit spaces .......... 357

Mark Mahowald, $b o$-resolutions . . . . . . . . . . . . . . . . . . . 365

Jan van Mill and Marcel Lodewijk Johanna van de Vel, Subbases, convex

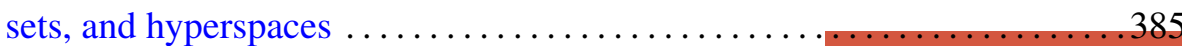

John F. Morrison, Approximations to real algebraic numbers by algebraic numbers of smaller degree $\ldots \ldots \ldots \ldots \ldots \ldots \ldots \ldots \ldots \ldots \ldots \ldots \ldots$

Caroline Series, An application of groupoid cohomology . . . . . . . . . . 415

Peter Frederick Stiller, Monodromy and invariants of elliptic surfaces . . . 433 Akihito Uchiyama, The factorization of $H^{p}$ on the space of homogeneous

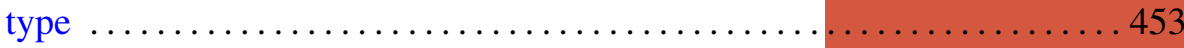

Warren James Wong, Maps on simple algebras preserving zero products.

II. Lie algebras of linear type 